MAGNETOHYDRODYNAMICS Vol. 54 (2018), No. 1-2, pp. 91-96

DOI: $10.22364 / \mathrm{mhd} .54 .1-2.16$

\title{
MODELLING OF ELECTRICAL CAPACITANCE DEPENDENCES OF MAGNETIC NANOFLUID LAYER IN AN ELECTRIC FIELD
}

\author{
M.S. Demin, T.F. Morozova \\ North-Caucasus Federal University, 1 Pushkin str., 355009 Stavropol, Russia
}

Results of the analysis of linear regression models are presented described in the experimental studies on the electrical capacity of a cell with a microlayer of magnetic nanofluid carried out with changed input parameters (factors), such as the polarizing voltage $(0-20 \mathrm{~V})$, the volume concentration of the disperse phase $(2.3-14.4 \%)$, the interelectrode gap $(100-200 \mu \mathrm{m})$, the layer temperature (293-368 K). Two-dimensional and three-dimensional graphical dependences of the electrical capacitance of the microlayer of magnetic nanofluid determined by the input parameters are presented.

Introduction. Previously performed numerous studies on microlayers of magnetic nanofluids (MNF) [1-4] have shown that the nonlinear effects are their distinctive feature, and the external electric field is a controlling factor of the structural state of the microlayer that appears under its action. Therefore, in order to obtain a controllable microstructure, it is necessary to develop a mathematical model of the structured microlayer with a dependence of its parameters on external influences. In this case, it is necessary to use the most simple mathematical models adequately describing the obtained experimental dependences.

To characterize complex systems with magnetic disperse systems, experimental and statistical methods are used which allow establishing relationships between the input parameters (factors) and output parameters (indicators of object functioning) in the form of regression equations. The MNF input parameters in the experiment were the following: the applied polarizing voltage $U_{\mathrm{P}}$ was varied from 0 to $20 \mathrm{~V}$, the interelectrode gap $d$ was varied from 0.05 to $2.0 \mathrm{~mm}$, the volume concentration of the disperse phase $\varphi$ was varied from 2.3 to $14.4 \%$, and the temperature of the magnetic nanofluid was varied 20 to $95^{\circ} \mathrm{C}$.

1. Experimental results and discussion. A microlayer of a magnetic nanofluid (magnetic iron in a kerosene-based nanofluid) with a thickness of 0.05$2 \mathrm{~mm}$ was confined between two plane-parallel electrodes with a size of $40 \times 50 \mathrm{~mm}^{2}$ (steel plates or glass plates with a conducting surface produced by depositing an $\mathrm{In}_{2} \mathrm{O}_{3} \mathrm{SnO}_{2}$ layer, i.e. a micro capacitor, were used).

The electrophysical parameters of microlayers of magnetic nanofluid were experimentally studied by the method discussed in [5], considering also the result obtained previously on the nonlinear dependence of the electrical capacitance $\Delta C / C=f\left(U_{\mathrm{P}}\right)$ in the polarizing voltage. These experiments have revealed that

- the position of the maximum of the electrical capacitance is determined by the interelectrode gap and by the concentration of the disperse phase (Figs. 1 and 2), with an increase in volume concentration of the disperse phase, with the interelectrode gap shifts of the position of the electrical capacitance maximum to the region of high electric field intensity; also, the decrease of the interelectrode gap to 0.1 and $0.05 \mathrm{~mm}$ contributes to the appearance of a more pronounced electrical capacitance maximum on the nonlinear dependence $\Delta C / C=f\left(U_{\mathrm{P}}\right)$ 
- an increase in temperature contributes to an increase of the electrical capacitance maximum (Figs. 3 and 4), whereas in the temperature range $20-65^{\circ} \mathrm{C}$ the maximum of the electrical capacitance becomes more pronounced and arises with the same value of the electric field for the given temperature range; the increase in temperature from 65 to $95^{\circ} \mathrm{C}$ causes the appearance of a maximum of electrical capacitance at a high electric field intensity.

In [6] it is suggested that the polarization in the MNF is due to two mechanisms: ionic and thermal orientational polarizations, and the experimentally obtained frequency dependences of the capacitance in [7] are described by a twocomponent fluid model with "fast" and "slow" charge carriers and, accordingly, by two polarization mechanisms.

An analysis and $3 \mathrm{D}$ plots of the dependences $C=f\left(T ; U_{\mathrm{P}}\right)$ and $C=f\left(\varphi ; U_{\mathrm{P}}\right)$ were added to the possible conclusions, i.e. from the entire concentration range under study, it is necessary to distinguish an MNF with a volume concentration of the dispersed phase of $6.3 \%$, for which the highest polarization and the crit-

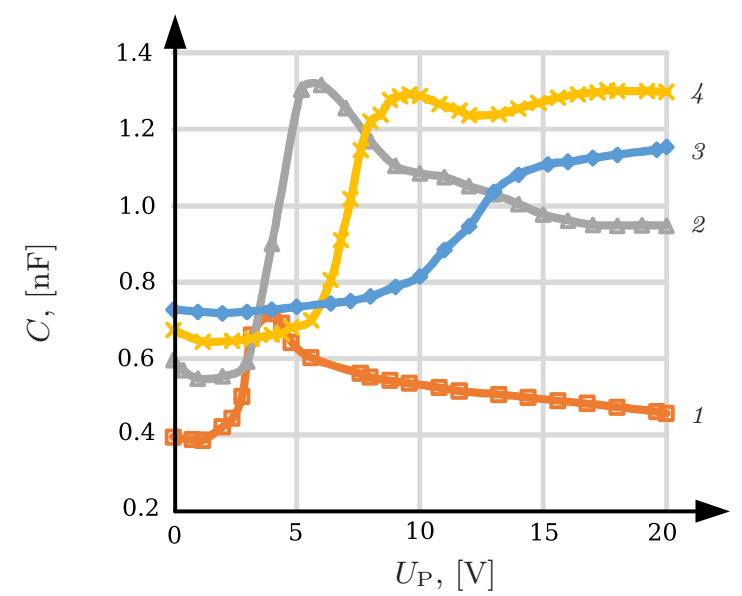

Fig. 1. Dependences $C=f\left(U_{\mathrm{P}}\right)$ of $100-\mu \mathrm{m}$-thick MNF layers with a temperature of $95^{\circ} \mathrm{C}$ with the following concentrations of solids: (1) $2.3 \%$, (2) $6.3 \%$, (3) $10.3 \%$ and (4) $14.4 \%$.

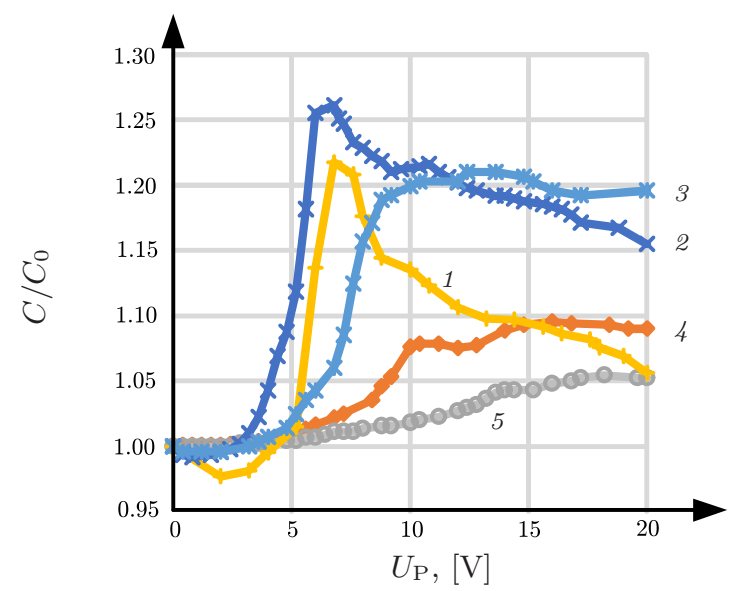

Fig. 2. Dependences $C / C_{0}=f\left(U_{\mathrm{P}}\right)$ of MNF layers with a temperature of $20^{\circ} \mathrm{C}$, $\varphi=6.3 \%$ with the following interelectrode gap $d$, mm: (1) 0.05 , (2) 0.1 , (3) 0.2 , (4) 1.0, (5) 2.0 . 
Modelling of electrical capacitance dependences of magnetic nanofluid layer...

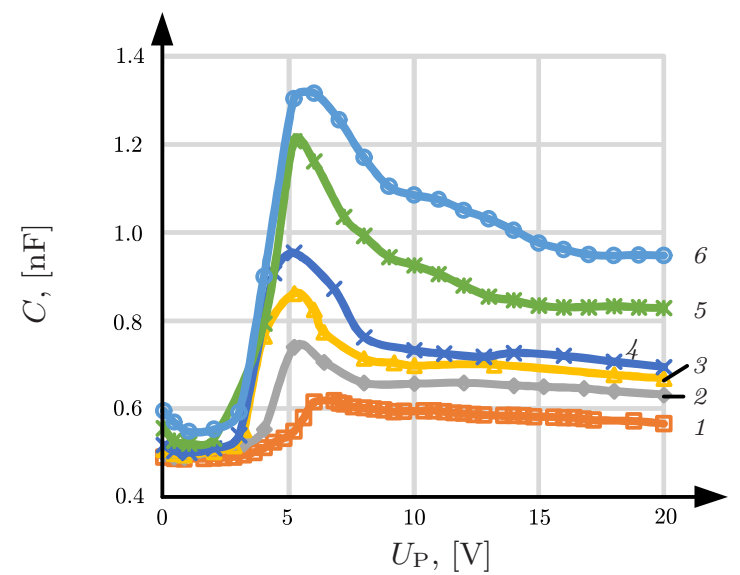

Fig. 3. Dependences $C=f\left(U_{\mathrm{P}}\right)$ of $100-\mu$ m-thick MNF layers with concentrations of solids of $\varphi=6.3 \%$ (a) and $\varphi=10.3 \%$ (b) with the following MNF temperatures: (1) $20^{\circ} \mathrm{C}$, (2) $45^{\circ} \mathrm{C}$, (3) $55^{\circ} \mathrm{C}$, (4) $65^{\circ} \mathrm{C}$, (5) $85^{\circ} \mathrm{C}$, (6) $95^{\circ} \mathrm{C}$.

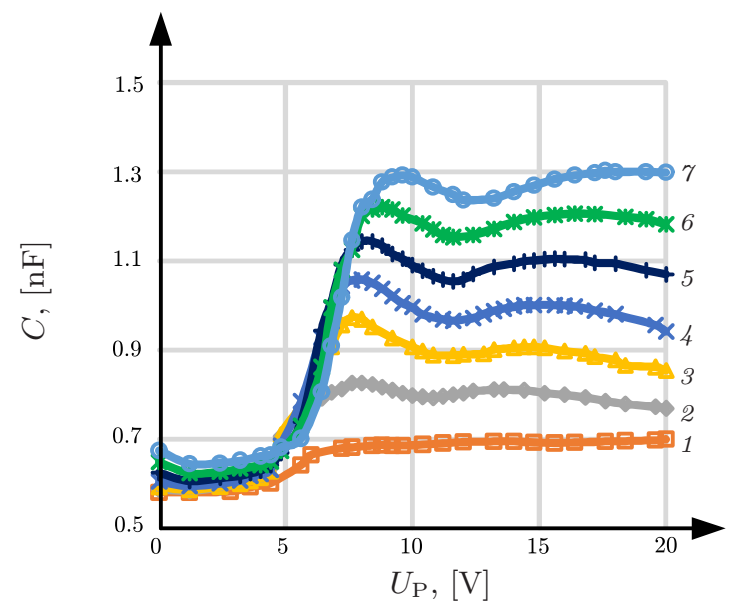

Fig. 4. Dependences $C=f\left(U_{\mathrm{P}}\right)$ of $100-\mu \mathrm{m}$-thick MNF layers with concentrations of solids of $\varphi=10.3 \%$ with the following MNF temperatures: (1) $20^{\circ} \mathrm{C}$, (2) $45^{\circ} \mathrm{C}$, (3) $55^{\circ} \mathrm{C}$, (4) $65^{\circ} \mathrm{C},(5) 75^{\circ} \mathrm{C},(6) 85^{\circ} \mathrm{C}$, (7) $95^{\circ} \mathrm{C}$.

ical temperature $65^{\circ} \mathrm{C}$ were revealed, at which a "splash" of the polarization was observed throughout the concentration range under investigation (Figs. 5 and 6).

To develop a mathematical model which could establish the relationships between the electrical capacitance of a cell with MNF and its parameters (factors), a software for statistical analysis of IBM SPSS Statistics was used.

2. Development of mathematical models. When building models in the IBM SPSS Statistics, the temperature from Celsius degrees was changed to the Kelvin thermodynamic temperature units and the coding factors were made at levels from -1 to +1 . With this program, estimates of the coefficients of the regression model and their standard errors in the $95 \%$ confidence interval were obtained. A linear model has been developed taking into account the effects of the interaction of the factors. According to the estimates, the Student's test was applied to the confidence level 0.95 to determine significant regression coefficients. The insignificant regression coefficients were neglected, and the significant ones were recalculated. As a result, a model has been developed for the entire range of 
variations of the polarizing voltage (from 0 to $20 \mathrm{~V}$ ):

$$
\begin{aligned}
C= & -777.021+67.143 \varphi+2190.186 d+4.513 T \\
& -0.161 \varphi T-3.863 d T-11.486 U \varphi \\
& +37.375 \varphi d U+0.044 \varphi T U-0.151 \varphi d T U
\end{aligned}
$$

allowing to consider average and maximum errors of $13.027 \%$ and $61.464 \%$, respectively, however, for practical purposes the obtained results are not applicable.

In $[6,7]$ it was found that the MNF has at least two polarization mechanisms. In addition, in [8] it was suggested that the occurrence of the first maximum of the electrical capacitance was due to the polarization of both the base ions and the dispersed phase particles, and the second maximum was initiated only by the polarization of the magnetic particles of the disperse phase.

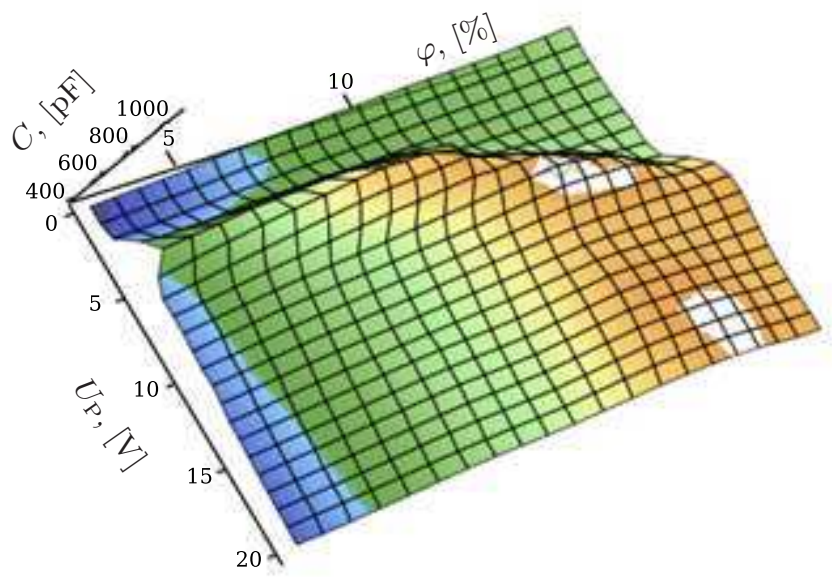

Fig. 5. Dependences $C=f\left(T ; U_{\mathrm{P}}\right)$ of $100-\mu$ m-thick MNF layers with a temperature of $\mathrm{MNF}$ of $65^{\circ} \mathrm{C}$.

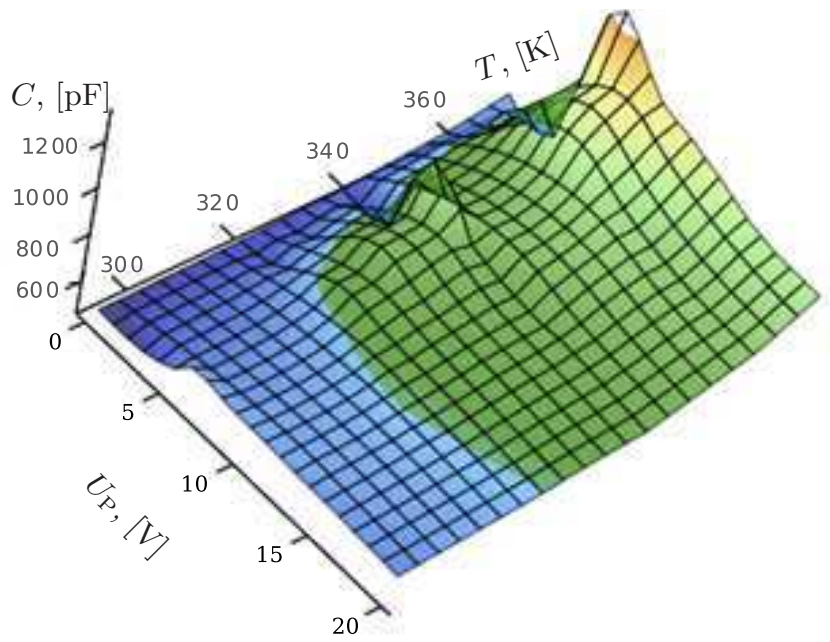

Fig. 6. Dependences $C=f\left(T ; U_{\mathrm{P}}\right)$ of $100-\mu$ m-thick MNF layers with a concentration of solids of $6.3 \%$. 
Modelling of electrical capacitance dependences of magnetic nanofluid layer...

Hence, the presence of two maxima of the electrical capacitance (Figs. 3 and 4 ) on the curves of the dependences $C=f\left(\varphi, U_{\mathrm{P}}, t^{\circ}\right)$ makes it reasonable to consider a model for two ranges of the polarizing voltage variations: from 0 to $10 \mathrm{~V}$ (Eq. (2)) and from 10 to $20 \mathrm{~V}$ (Eq. (3)), for which the linear regression models were developed taking into account the effects of the interaction of the factors:

$$
\begin{aligned}
C= & 72.251+82.538 \varphi-330.937 U+1.618 T-309.084 \varphi d \\
& -0.203 \varphi T-6.338 d T+4.308 U \varphi+1255.383 U d \\
& +1.055 U T+0.908 \varphi d T-3.789 d T U-0.077 \varphi d T U ; \\
C=- & 2940.356+145.865 \varphi+9949.851 d+156.873 U+11.167 T \\
- & 267.769 \varphi d-0.268 \varphi T-35.766 d T-18.103 U \varphi \\
- & 544.553 U d-0.523 U T+58.313 \varphi d U \\
+ & 0.058 \varphi T U+1.786 d T U-0.181 \varphi d T U .
\end{aligned}
$$

The models Eqs. (2) and (3) make it possible to obtain average errors of approximations of $10.817 \%$ and $8.695 \%$, respectively, for the first and for the second range.

3. Conclusions. The analysis of the developed models shows that the best approximation of the experimental data can be obtained from the models which consider the effects of the interaction of the factors for two intervals of variations of the polarizing voltage $(0-10 \mathrm{~V}$ and $10-20 \mathrm{~V})$, where the average approximation error was reduced to $10.817 \%$ and $8.695 \%$, respectively.

It follows from the analysis that for a more accurate description of the dependence of the electrical capacitance of a cell with a magnetic nanofluid on the microlayer thickness, on the medium temperature, on the magnitude of the polarizing voltage, and on the volume concentration of the disperse phase, it is necessary to use nonlinear regression models.

\section{References}

[1] V.M. Kozhevnikov And T.F. Morozova. Dielectric permittivity of a magnetic fluid stratum in electric and magnetic fields. Magnetohydrodynamics, vol. 37 (2001), no. 4, pp. 383-388.

[2] P.V. Averyanov, V.M. Kozhevnikov and T.F. Morozova. Electrophysical properties of a magnetodielectric colloid under the action of polarizing voltage. Izvestiya VUZov, Severo-Kavkazskiy region. Tekhn. Nauki, no. 1 (2004), pp. 49-54 (in Russian).

[3] T.F. Morozova. Electrophysical properties of a magnetodielectric colloid under the action of polarizing voltage. Sb. Nauch. Tr. Sev.-Kavk. Gos. Tekh. Univ., Ser. Fiz.-Khim., Stavropol, issue 5 (2001), pp. 79-85 (in Russian).

[4] T.F. Morozova, M.S. Demin. Structuring of a microlayer of a magnetic fluid with a different dispersion medium and a surfactant. Vestn. Sev.-Kavk. Gos. Tekh. Univ., no. 1 (2012), pp. 35-40 (in Russian).

[5] T.F. Morozova. Processes of polarization in a magnetic liquid microlayer. Vestn. Sev.-Kavk. Gos. Tekh. Univ., no. 2 (2010), pp. 45-50 (in Russian).

[6] T.F. Morozova, M.S. Demin. Analysis of the interrelation between the processes of polarization and microstructuring in a magnetic fluid layer. Technical Physics, vol. 87 (2017), no. 2, pp. 286-293. 
[7] V.M. Kozhevnikov, Yu.A. Larionov, M.S. Demin. Electrokinetic parameters of magnetodielectric colloid in nonstationary modes under the action of electric and magnetic fields. Vestn. Sev.-Kavk. Gos. Tekh. Univ., no. 1 (2007), pp. 56-61 (in Russian).

[8] V.M. Kozhevnikov, T.F. Morozova. Electrophysical Parameters of Thin Layers of Magnetic fluid and Its Components. Sb. Nauch. Tr. Sev.-Kavk. Gos. Tekh. Univ., Ser. Fiz.-Khim., Stavropol, issue 3 (1999), pp. 60-66 (in Russian).

Received 27.12.2017 